\title{
Renormalized sextic coupling constant for the two-dimensional Ising model from field theory
}

\author{
A. I. Sokolov and E. V. Orlov \\ Department of Physical Electronics, \\ Saint Petersburg Electrotechnical University, \\ Professor Popov Street 5, St. Petersburg, 197376, Russia
}

\begin{abstract}
The field-theoretical renormalization group approach is used to estimate the universal critical value $g_{6}^{*}$ of renormalized sextic coupling constant for the two-dimensional Ising model. A four-loop perturbative expansion for $g_{6}$ is calculated and resummed by means of the Padé-Borel-Leroy technique. Under the optimal value of the shift parameter $b$, providing the fastest convergence of the iteration procedure the estimates $g_{6}^{*}=1.10, g_{6}^{*} / g_{4}^{* 2}=2.94$ are obtained, which agree quite well with those deduced recently by Zinn, Lai, and Fisher [Phys. Rev. E 54, 1176 (1996)] from the high-temperature expansions.
\end{abstract}

PACS numbers: 05.70.Jk, 11.10.Gh, 64.60.Ak, 64.60.Fr 


\section{INTRODUCTION}

The two-dimensional (2D) Ising model in a zero magnetic field was exactly solved by L. Onsager more than 50 years ago. Since then, many attempts were made to develope a theory describing the behavior of this model under an external field but, nevertheless, an exact analytical description of its magnetized state has not been given up to now. On the other hand, for decades the critical behavior of various systems in ordering fields has attracted constant attention, being of prime interest both for theorists and experimentalists. Recently, the free energy (effective action) and, in particular, higher-order renormalized coupling constants $g_{2 k}$ for the basic models of phase transitions, have become the target of intensive theoretical studies [1-14]. These constants are related to the non-linear susceptibilities $\chi_{2 k}$, which enter the scaling equation of state and thus play very important role at criticality. Along with critical exponents and critical amplitude ratios, they are universal, i.e., possess, under $T \rightarrow T_{c}$, numerical values that are not sensitive to the physical nature of phase transition, depending only on the system dimensionality and the symmetry of the order parameter.

Calculation of the universal critical values of $g_{6}, g_{8}$, etc. for three-dimensional Ising and $O(n)$-symmetric models by a number of analytical and numerical methods showed that the field-theoretical renormalization group $(\mathrm{RG})$ approach in fixed dimensions yields most accurate numerical estimates for these quantities. It is a consequence of a rapid convergence of the iteration schemes originating from renormalized perturbation theory. Indeed, the resummation of four- and five-loop RG expansions by means of the Borel-transformationbased procedures gave the values for $g_{6}^{*}$, which differ from each other by less than 0.5\% [8,9] while the use of resummed three-loop RG expansion enabled one to achieve an apparent accuracy no worse than $1.6 \%$ [7, 13].

The field-theoretical RG approach, being very effective in 3D (see, e.g., Refs. [15] [17, [13]) is also known to be powerful in two dimensions. Properly resummed four-loop RG expansions lead to fair numerical estimates for critical exponents of a 2D Ising model [15] and give reasonable results for its random counterpart [18,19. It is natural, therefore, to use the field theory for the calculation of renormalized higher-order coupling constants of a 2D Ising model. In this paper, the $2 \mathrm{D}$ RG expansion for renormalized coupling constant $g_{6}$ will be calculated, and the numerical estimate for its universal critical value $g_{6}^{*}$ will be obtained.

\section{RG EXPANSION FOR SEXTIC COUPLING CONSTANT}

Within the field-theoretical language, the two-dimensional Ising model in the critical region is described by Euclidean scalar field theory with the Hamiltonian

$$
H=\int d^{2} x\left[\frac{1}{2} m_{0}^{2} \varphi^{2}+\frac{1}{2}(\nabla \varphi)^{2}+\lambda \varphi^{4}\right],
$$

where a bare mass squared $m_{0}^{2}$ is proportional to $T-T_{c}^{(0)}, T_{c}^{(0)}$ being the phase transition temperature in the absence of the order-parameter fluctuations. Taking fluctuations into account results in renormalizations of the mass $m_{0} \rightarrow m$, the field $\varphi=\varphi_{R} \sqrt{Z}$, and the coupling constant $\lambda=m^{2} Z_{4} Z^{-2} g_{4}$. Moreover, thermal fluctuations give rise to many-point 
correlations $<\varphi\left(x_{1}\right) \varphi\left(x_{2}\right) \ldots \varphi\left(x_{2 k}\right)>$ and, correspondingly, to higher-order terms in the expansion of the free energy in powers of magnetization $M$ :

$$
F(M)-F(0)=m^{2}\left[\frac{1}{2} \frac{M^{2}}{Z}+g_{4}\left(\frac{M^{2}}{Z}\right)^{2}+\sum_{k=3}^{\infty} g_{2 k}\left(\frac{M^{2}}{Z}\right)^{k}\right] .
$$

In the critical region, where fluctuations are so strong that they completely screen out the initial (bare) interaction, the behavior of the system becomes universal and dimensionless effective couplings $g_{2 k}$ approach their asymptotic limits $g_{2 k}^{*}$.

In order to estimate $g_{6}^{*}$, we will calculate RG expansion for $g_{6}$ and then apply Padé-BorelLeroy resummation technique to get proper numerical results. Accurate enough numerical estimates, as is well known, may be extracted only from a sufficiently long RG series. Below we will find the expression for $g_{6}$ in the four-loop approximation that will be shown to provide an interesting amount of fair numerical estimates.

The method of calculating the RG series that we use here is straightforward. Since in two dimensions higher-order bare couplings are believed to be irrelevant in RG sense, renormalized perturbative series for $g_{6}$ can be obtained from a conventional Feynman graph expansion of this quantity in terms of the only bare coupling constant $-\lambda$. In its turn, $\lambda$ may be expressed perturbatively as a function of renormalized dimensionless quartic coupling constant $g_{4}$. Substituting corresponding power series for $\lambda$ into original expansion, we can obtain the RG series for $g_{6}$. As was recently shown [5, 0, 8] the one-, two-, three- and fourloop contributions are formed by 1, 3, 16, and 94 one-particle irreducible Feynman graphs, respectively. Their calculation gives:

$$
g_{6}=\frac{36}{\pi}\left(\frac{\lambda Z^{2}}{m^{2}}\right)^{3}\left[1-11.817855 \frac{\lambda Z^{2}}{m^{2}}+110.37270\left(\frac{\lambda Z^{2}}{m^{2}}\right)^{2}-985.575\left(\frac{\lambda Z^{2}}{m^{2}}\right)^{3}\right],
$$

The perturbative expansion for $\lambda$ emerges directly from the normalizing condition $\lambda=$ $m^{2} Z_{4} Z^{-2} g_{4}$ and the well-known expansion for $Z_{4}$ [15]:

$$
Z_{4}=1+\frac{9}{\pi} g_{4}+5.1288114 g_{4}^{2}+10.511670 g_{4}^{3}+O\left(g_{4}^{4}\right)
$$

Combining these expressions we obtain

$$
g_{6}=\frac{36}{\pi} g_{4}^{3}\left(1-3.2234882 g_{4}+14.957539 g_{4}^{2}-85.7810 g_{4}^{3}\right) .
$$

This series will be used for estimation of the universal number $g_{6}^{*}$.

\section{RESUMMATION AND NUMERICAL ESTIMATES}

Being a field-theoretical perturbative expansion the series (5) has factorially growing coefficients, i.e., it is divergent (asymptotic). Hence, direct substitution of the fixed point value $g_{4}^{*}$ into (5) would not lead to satisfactory results. To get reasonable numerical estimate for $g_{6}^{*}$, some procedure making this expansion convergent should be applied. As is well known, the Borel-Leroy transformation 


$$
f(x)=\sum_{i=0}^{\infty} c_{i} x^{i}=\int_{0}^{\infty} t^{b} e^{-t} F(x t) d t, \quad F(y)=\sum_{i=0}^{\infty} \frac{c_{i}}{(i+b) !} y^{i} .
$$

diminishing the coefficients by the factor $(i+b)$ !, can play a role of such a procedure. Since the RG series considered turns out to be alternating, the analytical continuation of the Borel transform may be then performed by using Padé approximants. With the four-loop expansion (5) in hand, we can construct three different Padé approximants: [2/1], [1/2], and [0/3]. To obtain proper approximation schemes, however, only diagonal [L/L] and neardiagonal Padé approximants should be employed [20]. That's why further we limit ourselves to approximants $[2 / 1]$ and [1/2]. Moreover, the diagonal Padé approximant $[1 / 1]$ will be also dealt with, although this corresponds, in fact, to the usage of the lower-order, three-loop $\mathrm{RG}$ approximation.

The algorithm of estimating $g_{6}^{*}$ that we use here is as follows. Since the Taylor expansion for the free energy contains as coefficients the ratios $R_{2 k}=g_{2 k} / g_{4}^{k-1}$, rather than the renormalized coupling constants themselves,

$$
F(z)-F(0)=\frac{m^{2}}{g_{4}}\left(\frac{z^{2}}{2}+z^{4}+R_{6} z^{6}+R_{8} z^{8}+\ldots\right), \quad z^{2}=\frac{g_{4} M^{2}}{Z}
$$

we work with the RG series for $R_{6}$. It is resummed in three different ways based on the Borel-Leroy transformation and the Padé approximants just mentioned. The Borel-Leroy integral is evaluated as a function of the parameter $b$ under $g_{4}=g_{4}^{*}$. For the fixed point coordinate the value, $g_{4}^{*}=0.6125$ (Refs. [6,21,22]) is adopted, which was extracted from lengthy high-temperature expansions and is believed to be the most accurate estimate for $g_{4}^{*}$ available today. The optimal value of $b$, providing the fastest convergence of the iteration scheme, is then determined. It is deduced from the condition that the Padé approximants employed should give, for $b=b_{o p t}$, the values of $R_{6}^{*}$, which are as close as possible to each other. Finally, the average over three estimates for $R_{6}^{*}$ is found and claimed to be a numerical value of this universal ratio.

The results of our calculations are presented in Table 1. As one can see, for $b=1.24$ all three working approximants lead to practically identical values of $R_{6}^{*}$. Hence, we conclude that for 2D Ising model at criticality

$$
R_{6}^{*}=2.94, \quad g_{6}^{*}=1.10
$$

How close to their exact counterparts may these numbers be? To clear up this point, let us discuss the sensitivity of numerical estimates given by RG expansion (5) to the type of resummation. The content of Table 1 implies that, among others, the results given by Padé approximant [2/1] turn out to be most strongly dependent on the parameter $b$. This situation resembles that of 3D $O(n)$-symmetric model where Padé approximants of $[L-1 / 1]$ type for the $\beta$-function and critical exponents lead to numerical estimates demonstrating appreciable variation with $b$, while for diagonal and near-diagonal approximants the dependence of the results on the shift parameter is practically absent [13,15, 17. In our case, Padé approximants $[1 / 1]$ and $[1 / 2]$ may be referred to as generating such "stable" approximations for $g_{6}^{*}$. Since for $b$, varying from 0 to 15 (i.e., for any reasonable $b$ ), the magnitude of $g_{6}^{*}$ averaged over 
these two approximations remains within the segment $(1.044,1.142)$, it is hardly believed that the values (8) can differ from the exact ones by more than $5 \%$.

Another way we propose to judge how accurate our numerical results are is based on the comparison of the values of $g_{6}^{*}$ given by four subsequent RG approximations available. While within the one-loop order we get $g_{6}^{*}=2.633$, which is obviously very bad estimate, taking into account of higher-order RG contributions to $g_{6}$ improves the situation markedly. Indeed, two-, three-, and four-loop RG series, when resummed by means of the Padé-Borel technique with use of "most stable" approximants [0/1], [1/1], and [1/2] yield for $g_{6}^{*}$ the values $0.981,1.129$, and 1.051, respectively. Since this set of numbers demonstrates an oscillatory convergence, one may expect that the exact value of renormalized sextic coupling constant lies between the higher-order three-loop and four-loop estimates. It means that the deviation of numbers (8) from the exact values would not exceed $5 \%$.

Thus, we see that the four-loop RG expansion for $g_{6}$ and elaborated approximation scheme lead to accurate enough numerical data. On the other hand, the above arguments, as is always the case when we deal with diverging series, should be interpreted as suggestive, i.e., they would help us to fix only an apparent accuracy. It is of prime importance therefore to compare our estimates with those obtained by other methods. Recently, S.-Y. Zinn, S.-N. Lai, and M. E. Fisher, analyzing the high temperature series for various 2D Ising lattices, found that $R_{6}^{*}=2.943 \pm 0.007$ [6]; an almost identical value was obtained later in Ref. [14]. Our result for $R_{6}^{*}$ is seen to be in a brilliant agreement with this number. Of course, the practical coincidence of the lattice and four-loop RG estimates is occasional, and cannot be considered as a manifestation of extremely high accuracy of the methods discussed. The closeness of these estimates to each other, however, unambiguously demonstrates the power of both approaches. Moreover, such a closeness shed light on the role of a singular contribution to $g_{6}$, which can not be found perturbatively: this contribution is seen to be numerically small.

It is instructive also to compare our results with those given by another field-theoretical approach - the famous $\epsilon$-expansion. Today, for the Ising systems, only three terms in the $\epsilon-$ expansion for $R_{6}$ are known [23]:

$$
R_{6}^{*}=2 \epsilon\left(1-\frac{10}{27} \epsilon+0.63795 \epsilon^{2}\right) .
$$

Let us apply a simple Padé-Borel procedure to this series as a whole and to the series in brackets, and then let $\epsilon=2$. We find $R_{6}^{*}=3.19$ and $R_{6}^{*}=3.12$, respectively, i.e., the numbers differ from our estimate by less that $9 \%$. Keeping in mind the lack of a small parameter, these values of $R_{6}^{*}$ may be referred to as consistent. We believe that the proper account for higher-order terms in the $\epsilon$-expansion for $R_{6}$ will make corresponding numerical estimates closer to those extracted from $2 \mathrm{D}$ RG and high-temperature series.

\section{CONCLUSION}

To summarize, we have calculated the four-loop RG expansion for the renormalized sextic coupling constant $g_{6}$ of the two-dimensional Ising model. Resummation of this series by the Padé-Borel-Leroy method has lead at criticality, under the optimal value of the parameter 
$b$, to the results $g_{6}^{*}=1.10, g_{6}^{*} / g_{4}^{* 2}=2.94$. Having analyzed the sensitivity of the RG estimates to the type of resummation procedure, and the character of their dependence on the order of the RG approximation, an apparent accuracy of these values has been argued to be no worse than 5\%. Comparison of the RG estimates with their counterparts given by other approaches has shown that they are in very good agreement with those deduced recently for 2D Ising lattices from high-temperature expansions, and consistent as well with the result given by the Padé-Borel resummed three-loop $\epsilon$-expansion for $R_{6}^{*}$.

Notes added in proof. (i) Apart from Refs. 1-14, the higher order coupling constants in $D$ dimensions were studied in Refs. 24 26]. We are grateful to Dr. S. Boettcher, who brought these papers to our attention. (ii) Very good agreement exists between the first number Eq. (8) and the estimate $R_{6}^{*}=2.95 \pm 0.03$ (Ref. 14) obtained recently by matching the $\epsilon$-expansion available with the exact results known for $D=1$ and $D=0$. It may be

considered as an extra argument in favor of the high effectiveness of the field theory in the problem duscussed.

\section{ACKNOWLEDGMENTS}

We thank B. N. Shalaev for interesting discussions and E. Vicari for sending the paper (Ref. 14). This work was supported by the Ministry of General and Professional Education of the Russian Federation under Grant No. 97-14.2-16. 


\section{REFERENCES}

[1] C. Bagnuls and C. Bervillier, Phys. Rev. B 41, 402 (1990).

[2] N. Tetradis, C. Wetterich, Nucl. Phys. B 422, 541 (1994).

[3] M. M. Tsypin, Phys. Rev. Lett. 73, 2015 (1994).

[4] T. Reisz, Phys. Lett. B 360, 77 (1995).

[5] A. I. Sokolov, Fizika Tverdogo Tela 38, 640 (1996) [Phys. Solid State 38, 354 (1996)].

[6] S.-Y. Zinn, S.-N. Lai, and M. E. Fisher, Phys. Rev. E 54, 1176 (1996).

[7] A. I. Sokolov, V. A. Ul'kov, and E. V. Orlov, J. Phys. Studies 1, 362 (1997), condmat/9803352.

[8] A. I. Sokolov, E. V. Orlov, V. A. Ul'kov, Phys. Lett. A 227, 255 (1997), condmat/9803357.

[9] R. Guida and J. Zinn-Justin, Nucl. Phys. B 489, 626 (1997), hep-th/9610223.

[10] T. R. Morris, Nucl. Phys. B 495, 477 (1997), hep-th/9612117.

[11] P. Butera and M. Comi, Phys. Rev. E 55, 6391 (1997).

[12] M. M. Tsypin, Phys. Rev. B 55, 8911 (1997).

[13] A. I. Sokolov, Fizika Tverdogo Tela 40, 1284 (1998) [Phys. Solid State 40, 1169 (1998)].

[14] A. Pelissetto and E. Vicari, Nucl. Phys. B 540, 639 (1999); cond-mat/9801098.

[15] G. A. Baker, B. G. Nickel, and D. I. Meiron, Phys. Rev. B 17, 1365 (1978).

[16] J. C. Le Guillou and J. Zinn-Justin, Phys. Rev. B 21, 3976 (1980).

[17] S. A. Antonenko and A. I. Sokolov, Phys. Rev. E 51, 1894 (1995), hep-th/9803264.

[18] I. O. Mayer, A. I. Sokolov and B. N. Shalayev, Ferroelectrics 95, 93 (1989).

[19] I. O. Mayer, J. Phys. A: Math. Gen. 22, 2815 (1989).

[20] G. A. Baker, Jr. and P. Graves-Morris. Padé Approximants (Addison-Wesley, Reading, MA, 1981).

[21] G. A. Baker, Jr., Phys. Rev. B 15, 1552 (1977).

[22] P. Butera and M. Comi, Phys. Rev. B 54, 15828 (1996).

[23] J. Zinn-Justin, Quantum Field Theory and Critical Phenomena (Clarendon Press, Oxford, 1989).

[24] C. M. Bender, S. Boettcher, and L. Lipatov, Phys. Rev. Lett. 68, 3674 (1992).

[25] C. M. Bender and S. Boettcher, Phys. Rev. D 48, 4919 (1993).

[26] C. M. Bender and S. Boettcher., Phys. Rev. D 51, 1875 (1995). 


\section{TABLES}

TABLE I. The values of $R_{6}^{*}$ obtained by means of the Padé-Borel-Leroy technique for various $b$ within three-loop (approximant [1/1]) and four-loop (approximants [1/2] and [2/1]) RG approximations. The estimate for $b=1$ in the middle line is absent because corresponding Padé approximant turnes out to be spoilt by a positive axis pole.

\begin{tabular}{ccccccccccc}
\hline \hline$b$ & 0 & 1 & 1.24 & 2 & 3 & 4 & 5 & 7 & 10 & 15 \\
\hline$[1 / 1]$ & 2.741 & 2.908 & 2.937 & 3.009 & 3.077 & 3.125 & 3.161 & 3.212 & 3.258 & 3.301 \\
\hline$[1 / 2]$ & 2.827 & - & 2.936 & 2.877 & 2.853 & 2.838 & 2.828 & 2.814 & 2.800 & 2.787 \\
\hline$[2 / 1]$ & 3.270 & 2.988 & 2.936 & 2.800 & 2.667 & 2.568 & 2.491 & 2.380 & 2.273 & 2.171 \\
\hline \hline
\end{tabular}

